\title{
A Micromethod for Measuring Neutrophil Candidacidal Activity in Neonates
}

\author{
RONALD OSEAS AND ROBERT I. LEHRER ${ }^{(16)}$ \\ Departments of Pediatrics and Medicine, Center for the Health Sciences, University of California, Los Angeles, \\ California, USA
}

\section{Summary}

We developed a micromethod, requiring but a few drops of blood, for measuring the ability of neutrophils to kill ingested Candida albicans. The neutrophils of 10 normal adults killed $\mathbf{2 6 . 2}$ $\pm 9.5 \%$ of ingested $C$. albicans in $2.5 \mathrm{hr}$ in a standard fungicidal assay, and $21.4 \pm 6.5 \%$ in our new micromodification. Neutrophils from 14 full term, healthy 30 - to 40 -hr-old infants, studied with the micromethod, killed $23.2 \pm 6.0 \%$ of ingested $C$. albicans. We conclude that the neutrophils of normal neonates are competent in this sphere of antimicrobial activity.

\section{Speculation}

Traditional methods of measuring the microbicidal activity of leukocytes are frequently unsuited for the study of neonates because they require considerable amounts of blood. Micromethods, such as the one herein described, may facilitate analysis of the host factors related to infections in newborns and infants.

Blood leukocytes constitute a major host defense mechanism against microbial infection. Quantitative bactericidal $(1,9)$ and candidacidal $(3,4)$ methods have facilitated delineation, in adults and children, of disorders wherein defective leukocyte microbicidal function underlies increased susceptibility to infections. Less information is available regarding the microbicidal activity of leukocytes from newborns and infants $(8,10,12,13,14)$, largely because of limitations on the quantities of blood obtainable.

In this communication, we describe a micromethod for measuring the candidacidal activity of neutrophils, compare it with previously described methods, and report its use to study the neutrophils of normal newborn infants.

\section{MATERIALS AND METHODS}

\section{SUBJECTS}

Candidacidal assays were conducted on leukocytes from 10 healthy adults by a previously described method (5) and the presently reported micromethod. After appropriate informed consent was obtained from the parents, we used the micromethod to study the leukocytes of 14 newborns. Newborns were 30-40 hr old and healthy at the time of testing. Capillary blood, taken from a heel, was collected into $100-\mu \mathrm{l}$, nonheparinized capillary tubes (Drummond Scientific, Broomall, PA) and immediately transferred into the tissue culture chambers wherein coagulation ensued.

\section{CANDIDA ALBICANS}

C. albicans, strain UC 820 , was grown in $50 \mathrm{ml}$ Sabouraud-2\% dextrose broth (Difco) for 72-120 hr, washed with distilled water, counted in a hemocytometer with $0.01 \%$ methylene blue as diluent, and suspended in Hanks' balanced salt solution (HBSS). At least
$97 \%$ of the yeast cells were viable, as shown by their ability to exclude methylene blue after incubation in HBSS containing $25 \%$ fresh frozen (-70 C) group AB normal serum for $60 \mathrm{~min}(3)$.

\section{CANDIDACIDAL ASSAYS}

The standard candidacidal assay was performed essentially as described by Lehrer (5). Briefly, $1 \times 10^{6}$ yeast phase organisms and an equal number of neutrophils (added as mixed peripheral leukocytes) were incubated together at $37^{\circ}$ in a final volume of 1 $\mathrm{ml}$ HBSS containing $25 \%$ fresh frozen $\mathrm{AB}$ serum. The tubes were incubated $2.5 \mathrm{hr}$ in air with rotation $(30 \mathrm{rpm})$. Duplicate slides were then prepared from each tube by adding, with a Pasteur pipette, 2 drops of the incubation mixture and 3 drops of HBSS containing $25 \% \mathrm{AB}$ serum to the sample chamber of a Cytocentrifuge (Shandon Manufacturing, Sewickley, PA) and the centrifuging for $5 \mathrm{~min}$ at $800 \mathrm{rpm}$. The slides were rapidly air dried, fixed in absolute methanol for $1 \mathrm{~min}$, rinsed, and stained for 20 min with a 1:10 dilution of Giemsa stain (Matheson, Coleman and Bell, Norwood, $\mathrm{OH}$ ) in water. Controls lacking either serum or leukocytes contained negligible numbers (less than $3 \%$ ) of nonviable Candida cell "ghosts."

\section{MICROASSAY}

We made a simple reusable template by drilling three holes, 8 $\mathrm{mm}$ in diameter, through a $25 \times 75 \times 10 \mathrm{~mm}$ block of lucite. A rubber gasket $(25 \times 75 \times 2 \mathrm{~mm})$ with holes that matched those in the lucite was also made. The templates and gaskets were placed on the cleaned (acid-washed and extensively rinsed) standard microscope slides and held in place with metal clamps (Boston clip no. 3, Hunt Manufacturing Co., Statesville, NC) obtained from a local stationery store. The assembly converted each slide into an economical three-place tissue culture chamber.

The micromethod, as a modification of the standard assay, required only $100 \mu \mathrm{l}$ of whole blood to permit duplicate assays. Approximately $50 \mu \mathrm{l}$ of whole blood was placed in each chamber of the template, covered with Parafilm (American Can Company, Neenah, WI) and placed in Petri dishes humidified with moist gauze. After incubation for $60 \mathrm{~min}$ at $37^{\circ}$, the blood clot was removed with a sterile, 23 -gauge needle. The chambers were gently washed once with HBSS to leave an adherent monolayer of leukocytes, predominantly neutrophils. To each chamber, we added a drop of HBSS (approximately $50 \mu \mathrm{l}$ ) and $50 \mu \mathrm{l}$ of a solution containing $1 \times 10^{6} / \mathrm{ml}$ yeast-phase Candida organisms in HBSS with $25 \%$ normal group AB serum. The chambers were resealed and incubated for $2.5 \mathrm{hr}$ at $37^{\circ}$. After incubation, we removed the template and gasket and rapidly air dried the slide, which was then fixed in absolute methanol for $1 \mathrm{~min}$, and stained with Giemsa for $20 \mathrm{~min}$.

Slides from both the standard and microassays were examined by light microscopy at 1000 -fold magnification and 200 consecutively encountered Candida within phagocytic neutrophils were classified as germinated yeasts, ungerminated yeasts with blue 


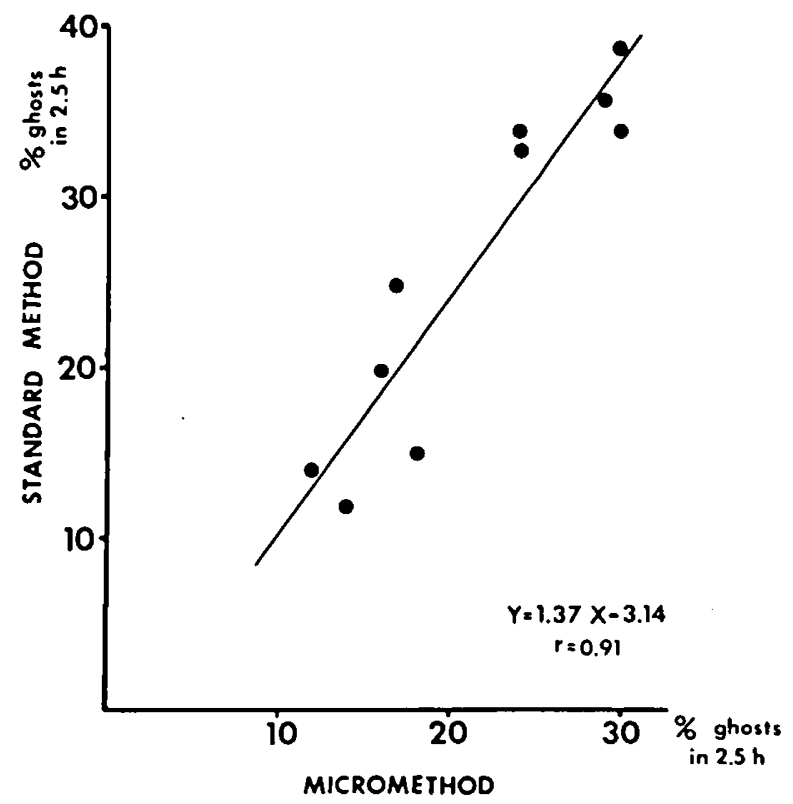

Fig. 1. Comparison of micromethod to standard assay. The percentage of Candida within neutrophils converted to ghosts after $2.5 \mathrm{hr}$ was determined in 10 healthy adults by the standard and microassays. Ghosts are yeast cells that have been killed and digested by the phagocyte, and reflect neutrophil candidacidal activity.

cytoplasm, or "ghosts." "Ghosts" were ungerminated yeasts whose cytoplasm did not stain blue (5), and represent organisms killed and degraded by the neutrophil. Viable cells maintained their blue cytoplasmic coloration and some developed filamentous pseudogerm tubes denoting their intracellular survival and growth.

\section{RESULTS}

Neutrophil leukocytes from 10 normal adults were examined by both the standard assay and the micromethod. By the standard assay, $26.2 \pm 9.5 \%$ (mean \pm SD) of Candida ingested by neutrophils were converted to ghosts by $2.5 \mathrm{hr}$. By the micromethod, $21.4 \pm 6.5 \%$ of $C$. albicans ingested by neutrophils were ghosts. The two measurements were highly correlated $(r=0.91$, Fig. 1).

Blood from 14 full-term healthy newborn infants was tested to measure their neutrophil candidacidal activity. After $2.5 \mathrm{hr}$ of incubation, $23.2 \pm 6.0 \%$ C. albicans within newborns' neutrophils were converted to ghosts. Thus, we found no significant difference between the candidacidal activity of adult or newborn neutrophils as measured by the micromethod.

\section{DISCUSSION}

C. albicans, a common inhabitant of our endogenous microbial flora, is a potential pathogen of man. Extensive investigations suggest that leukocyte function is a major host defense mechanism. Although plasma or serum do not kill $C$. albicans even though they contain antibodies and complement components, neutrophils, monocytes, and eosinophils can ingest and kill the organism (5-7).

Previous studies of the candidacidal activity of newborn infant leukocytes are contradictory $(10,14)$. Xanthou et al. reported their killing of ingested $C$. albicans to be markedly deficient (14), whereas Quie and Chilgren (10) found killing to be normal. Our study indicates that healthy newborn infants have normal candidacidal activity, and confirms the report of Quie and Chilgren.

Limitations in obtaining samples of newborns' blood adequate for standard methods of studying leukocyte function stimulated us to develop this microassay. Schmid and Brune (11) described a microassay adapted from the dye exclusion technique of Lehrer and Cline (3). Their assay also reduced the amount of blood needed, but required prompt examination of inconvenient wet mount preparations containing leukocytes and methylene blue. Moreover, as methylene blue improves the in vitro candidacidal activity of neutrophils from patients with chronic granulomatous disease (2), their method may be prone to artifactual distortion under certain conditions.

Our current modification is inexpensive, simple to perform, requires but a few drops of blood, and provides a permanent slide record. We found it to correlate well with our previous assays of candidacidal activity, and believe it could be a useful screening test for early detection of functional neutrophil disorders in neonates and infants.

\section{REFERENCES AND NOTES}

1. Dossett. J. H., Williams, R. C., Jr.. and Quie, P. G.: Studies on interaction of bacteria, serum factors and polymorphonuclear leukocytes in mothers and newborns. Pediatrics, 44: 49 (1969).

2. Lehrer, R. I.: Defective candidacidal activity in chronic granulomatous disease neutrophils: Correction by redox dyes. Clin. Res., 17: 331, (1969).

3. Lehrer, R. I., and Cline, M. J.: Interaction of Candida albicans with human leukocytes and serum. J. Bacteriol.. 98: 996 (1969).

4. Lehrer, R. I., and Cline, M. J.: Leukocyte myeloperoxidase deficiency and disseminated candidiasis: The role of myeloperoxidase in resistance to Candida infection. J. Clin. Invest., 48: 1478 (1969).

5. Lehrer, R. I.: Measurement of candidacidal activity of specific leukocyte types in mixed cell populations. I. Normal. myeloperoxidase-deficient. and chronic granulomatous disease neutrophils. Infect. Immunol.. 2: 42 (1970).

6. Lehrer, R. I.: Measurement of candidacidal activity of specific leukocyte types in mixed cell populations. II. Normal and chronic granulomatous disease eosinophils. Infect. Immunol., 3: 800 (1971).

7. Lehrer, R. I.: The fungicidal mechanisms of human monocytes. I. Evidence for myeloperoxidase-linked and myeloperoxidase-independent candidacidal mechanisms. J. Clin. Invest., 55: 338 (1975).

8. Orlowski, J. P., Sieger, L., and Anthony, B. F.: Bactericidal capacity of newborn infants. J. Pediat., 89: 797 (1976).

9. Quie, P. G., White, J. G., Holmes, B., and Good, R. A.: In vitro bactericidal capacity of human polymorphonuclear leukocytes: Diminished activity in chronic granulomatous disease of childhood. J. Clin. Invest., 46: 668 (1967).

10. Quic. P. G., and Chilgren. R. A.: Acute disseminated and chronic mucocutaneous candidiasis. Semin. Hematol., 8: 227 (1971).

11. Schmid. L., and Brune. K.: Assessment of phagocytic and antimicrobial activity of human granulocytes. Infect. Immunol. 10: 1120 (1974).

12. Weston, W. L.. Carson, B. S., Barkin. R. M.. and Slater. G. E.: Monocytemacrophage function in the newborn. Clin. Res., 24: 180A (1976).

13. Wright. W. C.. Jr.. Ank, B., Herbert. J., and Stiehm, E. R.: Impaired antibacterial activity of leukocytes of sick newborns. Pediatrics, 56: 579 (1975).

14. Xanthou, M., Valassi-Adam, E., Kintzonidou, E., and Matsaniotis, N.: Phagocytosis and killing activity of Candida albicans by blood leukocytes of healthy term and preterm babies. Arch. Dis. Childhood, 50: 72 (1975).

15. This research was supported in part by Research Grants AI 12171. CA 15688. and RR 5354 from the USPHS and by an award from the Karen Brigham Memorial Fund.

16. Correspondence should be addressed to: Dr. Robert I. Lehrer, Department of Medicine, Centre for Health Sciences. University of California. School of Medicine, Los Angeles, CA 90024 (USA).

17. Received for publication August 12. 1977.

18. Accepted for publication October 18, 1977. 